\title{
Comparison of short-term outcomes between minimally invasive McKeown and Ivor Lewis esophagectomy for esophageal or junctional cancer: a systematic review and meta-analysis
}

This article was published in the following Dove Press journal: OncoTargets and Therapy

\author{
Jianqing Deng ${ }^{1, *}$ \\ Qingqing $\mathrm{Su}^{2, *}$ \\ Zhipeng Ren' \\ Jiaxin Wen' \\ Zhiqiang Xue \\ Lianbin Zhang' \\ Xiangyang Chu' \\ 'Department of Thoracic Surgery, \\ Chinese PLA General Hospital, \\ Beijing, People's Republic of China; \\ ${ }^{2}$ Department of Nursing Department, \\ Chinese PLA General Hospital, \\ Beijing, People's Republic of China \\ *These authors contributed equally \\ to this work
}

Correspondence: Xiangyang Chu Department of Thoracic Surgery, Chinese PLA General Hospital, 28 Fuxing Road, Haidian District, Beijing 100853, People's Republic of China

Tel +86734 3090236

Fax +867343090236

Email drchu30I@aliyun.com
Purpose: Minimally invasive esophagectomy is increasingly performed for esophageal or gastroesophageal junctional cancer, with advantages of improved perioperative outcomes in comparison with open esophagectomy. McKeown and Ivor Lewis are widely used procedures of minimally invasive esophagectomy, and there have been controversies on which one is preferred for patients with resectable esophageal or junctional cancer.

Patients and methods: This review was registered at the International Prospective Register of Systematic Reviews (number CRD42017075989). Studies in PubMed, Embase, Web of Science, the Cochrane Library, and ClinicalTrials.gov were thoroughly investigated. Eligible studies included prospective and retrospective studies evaluating short-term outcomes of minimally invasive McKeown esophagectomy (MIME) vs minimally invasive Ivor Lewis esophagectomy (MILE) in patients with resectable esophageal or junctional tumors. Main parameters included anastomotic leak and 30-day/in-hospital mortality. Overall incidence rates (ORs)/weighted mean difference (WMD) with $95 \%$ confidence intervals (CIs) were calculated by employing random-effects models.

Results: Fourteen studies containing 3,468 cases were included in this meta-analysis. Age, male sex, and American Joint Committee on Cancer (AJCC) stage between the 2 groups were not statistically different. MIME led to more blood loss, longer operating time, and longer hospital stay than MILE. MIME was associated with higher incidence of pulmonary complications ( $\mathrm{OR}=1.96,95 \% \mathrm{CI}=1.28-3.00)$ as well as total anastomotic leak $(\mathrm{OR}=2.55$, $95 \% \mathrm{CI}=1.40-4.63)$, stricture $(\mathrm{OR}=2.07,95 \% \mathrm{CI}=1.05-4.07)$, and vocal cord injury/palsy $(\mathrm{OR}=5.62,95 \% \mathrm{CI}=3.46-9.14)$. In addition, the differences of $\mathrm{R} 0$ resection rate, number of lymph modes retrieved, blood transfusion rate, length of intensive care unit stay, incidence of cardiac arrhythmia, and Chyle leak between MIME and MILE were not statistically significant. Notably, incidence of severe anastomotic leak ( $\mathrm{OR}=1.28,95 \% \mathrm{CI}=0.73-2.24)$ and 30-day/ in-hospital mortality $(\mathrm{OR}=1.76,95 \% \mathrm{CI}=0.92-3.36)$ as well as 90 -day mortality $(\mathrm{OR}=2.22$, $95 \% \mathrm{CI}=0.71-6.98$ ) between the 2 procedures were also not significantly different.

Conclusion: This study suggests that MIME and MILE are comparable with respect to clinical safety. MILE may be a better option when oncologically and clinically suitable. MIME is still a safe alternative procedure when clinically indicated. However, this evidence is at risk for bias; randomized controlled trials are needed to validate or correct our results.

Keywords: Ivor Lewis esophagectomy, McKeown esophagectomy, minimally invasive esophagectomy (MIE), cervical anastomosis, intrathoracic anastomosis 


\section{Introduction}

Esophageal cancer has rapidly become a global concern. Its incidence increased by $44 \%$ in the past 2 decades, from 316,000 patients diagnosed in 1990 to 455,800 new cases estimated in 2012. ${ }^{1,2}$ Despite the advancement of medical techniques, esophageal cancer still has a poor 5-year survival rate that varies between $15 \%$ and $25 \%$ in most countries. ${ }^{3}$ Radical esophagectomy with adequate lymphadenectomy, sometimes after neoadjuvant therapy, is the main option for treating esophageal cancer. ${ }^{4}$

Minimally invasive esophagectomy (MIE) is being increasingly implemented because it has been proved to be superior for perioperative outcomes and possesses equal survival benefit compared with open esophagectomy. 5,6 MIE consists of McKeown, Ivor Lewis, and transhiatal procedures. ${ }^{7}$ Surgeons prefer the transthoracic MIE procedures (McKeown and Ivor Lewis), because they can make adequate thoracic lymph node dissection. ${ }^{8}$ However, the superiority of one procedure over the other continues to be controversial. ${ }^{9,10}$

A previous meta-analysis compared short-term outcomes between minimally invasive McKeown esophagectomy (MIME) and minimally invasive Ivor Lewis esophagectomy (MILE) for esophageal cancer, ${ }^{8}$ however, it ignored preoperative clinical data and other relevant literature in People's Republic of China where new esophageal cancer cases account for about $50 \%$ of the cases worldwide annually. ${ }^{11}$ In addition, it might not be proper to make a pooled analysis by combining the severe anastomotic leak in the biggest studies (1,011 cases) with total anastomotic leak in the other 4 studies (648 cases totally) to get the overall anastomotic leak rate in a meta-analysis. We, therefore, performed a more comprehensive meta-analysis involving the Chinese patients and other latest relevant publications.

\section{Materials and methods}

All procedures of this review were guided by Cochrane Handbook for Systematic Reviews of Interventions Version 5.1.0, and this meta-analysis was reported according to the Preferred Reporting Items for Systematic Reviews and Meta-Analyses (PRISMA) Statement and was registered at the International Prospective Register of Systematic Reviews (number CRD42017075989). ${ }^{12,13}$

\section{Searching strategy}

PubMed, Embase, Science Citation Index, The Cochrane Library, and ClinicalTrials.gov were thoroughly searched with the following terms or combinations of these terms (as either key words or MeSH terms): "Minimally Invasive esophagectomy/oesophagectomy" or "laparothoracoscop esophagectomy," "thoracolaparoscop esophagectomy," "video assisted thoracic surgery (VATS) Esophagectomy," "video-assisted esophagectomy" or "Minimally Invasive McKeown esophagectomy" or "Minimally Invasive Ivor Lewis esophagectomy" (date to March 2018). Following the initial screening based on abstracts, we obtained full texts of candidate publications and examined the reference lists of each retrieved article carefully in order to identify additional potentially relevant articles.

\section{Selections of studies}

All searching records were screened on title and abstract by the 2 authors (Deng and Su) independently. All studies that were not excluded in the screening stage were further assessed in full text for eligibility. If discrepancies occurred, discussion with a third author (Chu) would be held to reach a consensus. This process will be described in a flowchart according to the PRISMA statement. ${ }^{13}$

\section{Inclusion criteria}

The major inclusion criteria were the following: 1) prospective and retrospective studies and 2) studies that compare short-term outcomes of MIME and MILE in patients with resectable esophageal or junctional tumors.

\section{Exclusion criteria}

The exclusion criteria were as follows: 1) studies that were not compared, 2) overlapped studies, and 3) studies that did not report main results such as mortality and anastomotic leak.

\section{Data extraction}

All data were extracted from article texts, tables, and figures by 2 investigators (Deng and $\mathrm{Su}$ ) independently. The following data were extracted: names of first authors, durations of studies, study design, countries, numbers of patients included, age, gender, tumor stage, location of cancer, as well as intraoperative and postoperative data and main complications including 30-day/in-hospital or 90-day mortality and anastomotic leak. If the 2 investigators had different ideas on any data, the third senior investigator (Chu) would be asked to check to reach consensus on the data.

\section{Quality assessment}

The quality of the included studies was evaluated by the Newcastle-Ottawa Quality Assessment Scale (NOS) for 
cohort studies. ${ }^{14}$ Each study was accessed separately by 2 independent reviewers (Ren and Wen). The NOS contains 3 sections: the selection of the involved groups, the comparability between the groups, and the assessment of follow-up and outcomes. The number of total stars was recorded to reflect the quality of the included studies, which ranged from 1 (lowest quality) to 9 (highest quality).

\section{Statistical analysis}

Meta-analysis was performed using the software Stata 12.0 (Stata Corp LP, College Station, TX, USA; 2011). Overall incidence rates (odds ratios; ORs)/weighted mean difference (WMD) with 95\% confidence intervals (CIs) were calculated for binary and continuous parameters, respectively, by employing random-effects models regardless of the heterogeneity, thereby obtaining conservative results. ${ }^{15}$ According to Cochrane Reviewer Handbook 5.1.0, if a study with a reasonably large sample size reports a parameter as a median and interquartile range where the central $50 \%$ of participants' outcomes lie for continuous data, the width of interquartile range will be approximately 1.35 standard deviations. ${ }^{12}$ So, the value of standard deviation can be obtained by dividing the interquartile range by 1.35 . Heterogeneity between studies was assessed using the Cochran $Q$ and the $I^{2}$ statistic. An $I^{2}$ value $>50 \%$ was considered substantial heterogeneity, and subgroup analysis or meta-regression analysis (when the number of studies included $\geq 10$ ) was performed to find out the potential origin of heterogeneity, if necessary. A 2 -sided $P$-value $<0.05$ was considered statistically significant. If the number of studies we had included was $<20$, Egger's test would be selected to reveal potential publication bias, because it is thought that if the number of included studies is $<20$, the Egger's test is more sensitive to find out potential publication bias than the Begg's test. ${ }^{15}$ If there were more than 20 studies, both tests would be performed. If this test indicated apparent publication bias $(P<0.1)$, the trim-and-fill computation was carried out to estimate the effect of publication bias on the interpretation of the results. Sensitivity analysis was performed using the leave-one-out approach.

\section{Results Study characteristics}

A flowchart of the literature searching process according to PRISMA is shown in Figure 1. With our searching strategy, we identified 2,610 publications after removing duplicates. After carefully reviewing their titles and abstracts, 20 records were potentially eligible for our review. Then, we assessed full-text articles and found that among them 2 studies were meta-analyses, ${ }^{8,16} 2$ studies were duplicates of previous studies, and 2 studies did not report main results. Finally, 3,468 patients from 14 cohort studies underwent totally minimally invasive McKeown or Ivor Lewis esophagectomy were subjected to this meta-analysis. ${ }^{9,10,17-28}$ No randomized controlled studies or studies adopting hybrid MIE were found. Major characteristics and NOS quality star of the included studies are summarized in Tables 1 and 2 (surgical details and other outcomes can be found in Supplementary materials). The results of our meta-analysis are shown in Table 3.

\section{Preoperative clinical data}

Ten studies reported age as mean (SD) or median (interquartile range) in the 2 groups; there was no statistical significance between the 2 groups after pooled analysis (WMD $=0.77$, $95 \% \mathrm{CI}=-0.21$ to $1.75, P=0.12$ ). Ten studies containing 2,598 cases reported the number of male cases in 2 groups; no apparent difference was detected (OR $=1.13$, 95\% $\mathrm{CI}=0.93-1.37, P=0.21$ ). AJCC staging (stages 0 , I, and II) of patients' esophageal cancer was reported in 7 studies with 1,132 cases; no statistical significance was found between the 2 groups $(\mathrm{OR}=0.87,95 \% \mathrm{CI}=0.63-1.22, P=0.42)$. Few studies reported the preoperative comorbidity and neoadjuvant therapy to provide meaningful pooled results.

\section{Intraoperative data}

Operating time was reported in 10 studies (1,666 cases). MIME was associated with significantly longer operating time $(\mathrm{WMD}=36.49,95 \% \mathrm{CI}=7.12-65.86, P=0.01)$. MIME was found to cause more intraoperative blood loss than MILE (WMD $=16.9,95 \% \mathrm{CI}=3.22-30.58, P=0.02$ ), but the difference of transfusion rate was of no statistical significance $(\mathrm{OR}=1.25,95 \% \mathrm{CI}=0.29-5.38, P=0.76)$. Seven studies with 2,419 cases reported the number of lymph nodes retrieved; there was no statistical significance between the 2 groups after pooled analysis (WMD $=3.22,95 \% \mathrm{CI}=-0.95$ to 7.40, $P=0.13$ ). The difference of $\mathrm{R} 0$ resection rate between the 2 groups was not statistically significant after pooled analysis of the data reported by 4 studies containing 1,687 cases $(\mathrm{OR}=1.34,95 \% \mathrm{CI}=0.36-5.02, P=0.67)$. Significant between-study heterogeneity was detected for operating time $\left(I^{2}=98.5 \%\right)$, blood loss $\left(I^{2}=73.2 \%\right)$, transfusion $\left(I^{2}=71.4 \%\right)$, and lymph nodes retrieved $\left(I^{2}=96.7 \%\right)$. Subgroup analysis according to a country (China/non-China) could not be performed for blood loss and transfusion because there was only 1 study in subgroup non-China. Such a subgroup 


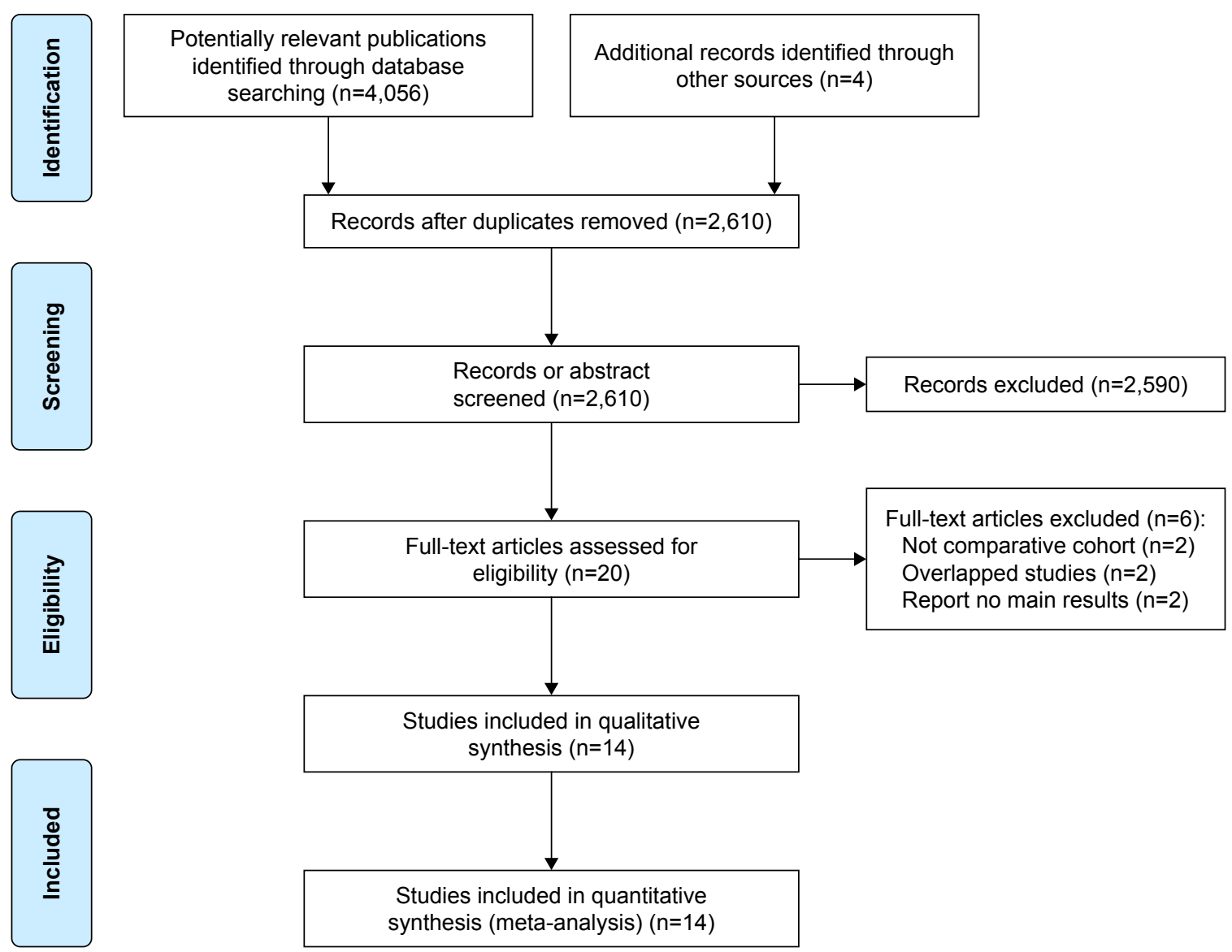

Figure I PRISMA 2009 flow diagram.

Abbreviation: PRISMA, Preferred Reporting Items for Systematic Reviews and Meta-Analyses.

analysis performed for lymph nodes retrieved showed that heterogeneity still existed in 2 groups. A RemL univariate meta-regression according to a country or publication year was performed to detect the potential origin of the heterogeneity of operating time, but neither of them was related to the heterogeneity statistically.

\section{Postoperative data}

Nine studies containing 2,439 patients reported the length of hospital stay. After pooled analysis, we found that MIME led to longer hospital stay $(\mathrm{WMD}=1.29,95 \% \mathrm{CI}=0.27-2.31$, $P=0.01)$. Four studies $(1,496$ cases $)$ reported the length of intensive care unit (ICU) stay, and no significant difference between the 2 groups was found (WMD $=-0.3,95 \%$ $\mathrm{CI}=-1.28$ to $0.67, P=0.54)$. We detected considerable heterogeneity for both postoperative outcome parameters $\left(I^{2}>50 \%\right)$. We could only perform subgroup analysis according to a country (China/non-China) for length of hospital stay, and heterogeneity only existed in the China subgroup $\left(I^{2}=88.0 \%\right)$.

\section{Complications}

Pulmonary and cardiac complications

Ten studies including 1,866 cases reported pulmonary complications, where $17.3 \%(177 / 1,026)$ were in the MIME group and $12.0 \%$ (101/840) were in the MILE group. There was evidence of reduced risk of pulmonary complications in the MILE group $(\mathrm{OR}=1.96,95 \% \mathrm{CI}=1.28-3.00, P=0.002)$. Five studies (824 cases) reported cardiac arrhythmia, and there was no significant difference between the MIME and MILE groups $(\mathrm{OR}=1.2,95 \% \mathrm{CI}=0.65-2.21, P=0.56)$. Moreover, no significant heterogeneity was found for pulmonary and cardiac complications.

\section{Anastomotic leak}

Thirteen studies including 2,457 cases reported the rates of anastomotic leak, where $12.9 \%(131 / 1,292)$ were in the MIME group and 5.7\% $(63 / 1,165)$ were in the MILE group; we found that MIME was associated with higher incidence of anastomotic leak than MILE (OR $=2.55,95 \% \mathrm{CI}=1.40-4.63$, $P=0.002)$ after pooled analysis, as shown in Figure 2. 


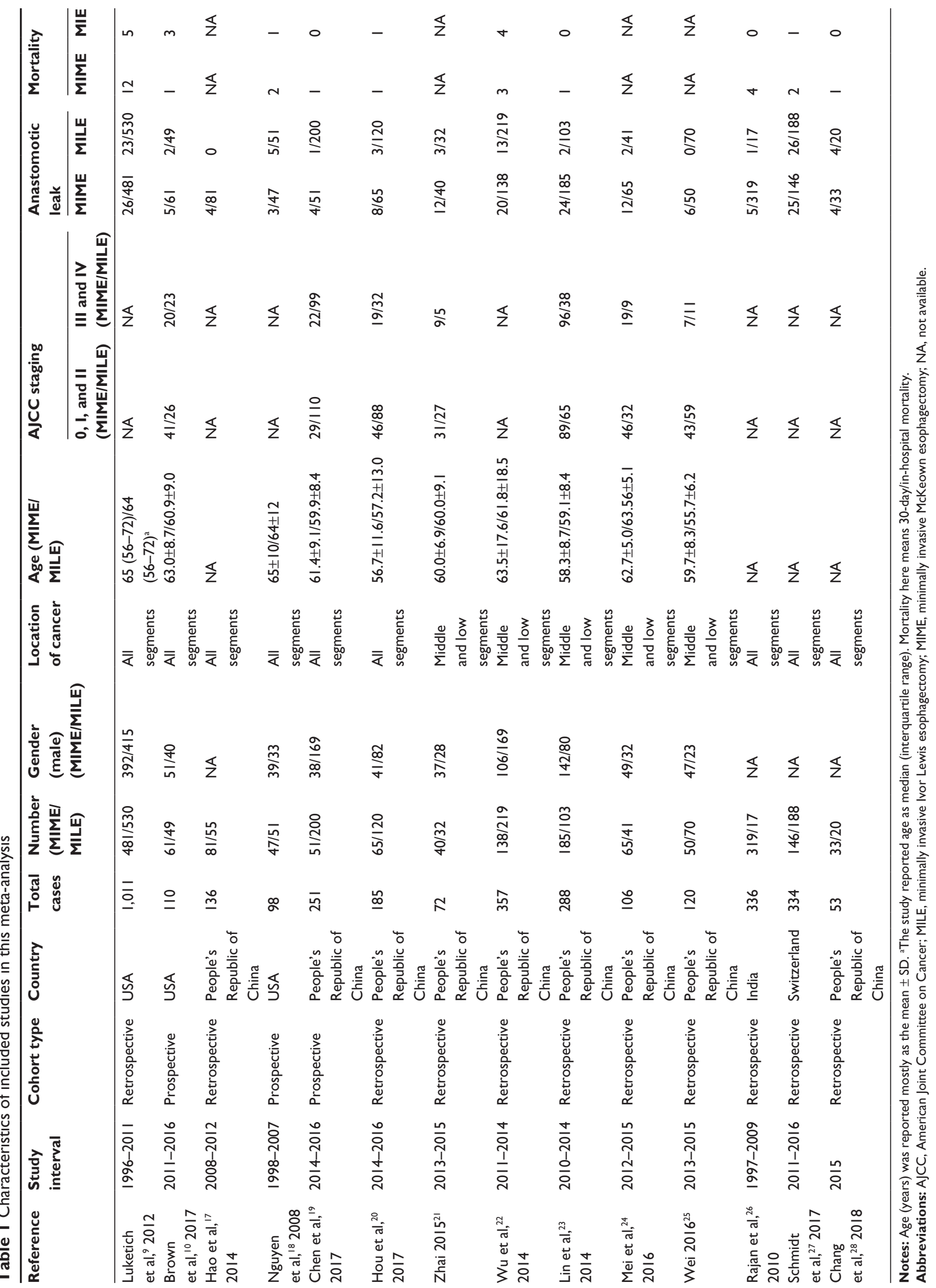


Table 2 NOS for risk of bias assessment of studies included in the meta-analysis

\begin{tabular}{|c|c|c|c|c|c|c|c|c|c|}
\hline \multirow[t]{2}{*}{ Reference } & \multicolumn{4}{|l|}{ Selection } & \multirow[t]{2}{*}{ Comparibility } & \multicolumn{3}{|l|}{ Outcome } & \multirow[t]{2}{*}{ Overall } \\
\hline & Representative & Selection & $\begin{array}{l}\text { Ascertainment } \\
\text { of exposure }\end{array}$ & Demonstration & & Outcome & Follow-up & $\begin{array}{l}\text { Adequacy } \\
\text { follow-up }\end{array}$ & \\
\hline $\begin{array}{l}\text { Luketich } \\
\text { et al, }{ }^{9} 2012\end{array}$ & $\star$ & $\star$ & $\star$ & $\star$ & is & $\star$ & $\star$ & $\star$ & 7 \\
\hline $\begin{array}{l}\text { Brown } \\
\text { et al, }{ }^{10} 2017\end{array}$ & $\star$ & $\star$ & $\star$ & $\star$ & $\star$ & $\star$ & $\star$ & $\star$ & 8 \\
\hline $\begin{array}{l}\text { Hao et al, }{ }^{17} \\
2014\end{array}$ & $\star$ & 访 & $\star$ & $\star$ & it & $\star$ & $\star$ & $\star$ & 6 \\
\hline $\begin{array}{l}\text { Nguyen } \\
\text { et al, }{ }^{18} 2008\end{array}$ & $\star$ & $\star$ & $\star$ & $\star$ & is & $\star$ & $\star$ & $\star$ & 7 \\
\hline $\begin{array}{l}\text { Chen et al, }{ }^{19} \\
2017\end{array}$ & $\star$ & $\star$ & $\star$ & $\star$ & is & $\star$ & $\star$ & $\star$ & 7 \\
\hline $\begin{array}{l}\text { Hou et al, }{ }^{20} \\
2017\end{array}$ & $\star$ & $\star$ & $\star$ & $\star$ & is & $\star$ & $\star$ & $\star$ & 7 \\
\hline Zhai $2015^{21}$ & $\star$ & $\star$ & $\star$ & $\star$ & $\star$ & $\star$ & $\star$ & $\star$ & 8 \\
\hline $\begin{array}{l}\text { Wu et al, }{ }^{22} \\
2014\end{array}$ & $\star$ & $\star$ & $\star$ & $\star$ & is & $\star$ & $\star$ & $\star$ & 7 \\
\hline $\begin{array}{l}\text { Lin et al, }{ }^{23} \\
2014\end{array}$ & $\star$ & $\star$ & $\star$ & $\star$ & is & $\star$ & $\star$ & $\star$ & 7 \\
\hline $\begin{array}{l}\text { Mei et al, }{ }^{24} \\
2016\end{array}$ & $\star$ & $\star$ & $\star$ & $\star$ & is & $\star$ & $\star$ & $\star$ & 7 \\
\hline Wei $2016^{25}$ & $\star$ & $\star$ & $\star$ & $\star$ & $\star$ & $\star$ & $\star$ & $\star$ & 8 \\
\hline $\begin{array}{l}\text { Rajan et al, }{ }^{26} \\
2010\end{array}$ & $\star$ & it & $\star$ & $\star$ & is & $\star$ & $\star$ & $\star$ & 6 \\
\hline $\begin{array}{l}\text { Schmidt } \\
\text { et al, }{ }^{27} 2017\end{array}$ & $\star$ & $\star$ & $\star$ & $\star$ & $\star$ & $\star$ & $\star$ & $\star$ & 8 \\
\hline $\begin{array}{l}\text { Chang et al, }{ }^{28} \\
2018\end{array}$ & $\star$ & $\star$ & $\star$ & $\star$ & is & $\star$ & $\star$ & $\star$ & 7 \\
\hline
\end{tabular}

Note: “ $\star$ ” means score one point, “攻” means score zero point.

Abbreviation: NOS, Newcastle-Ottawa Scale.

High heterogeneity was detected among studies $\left(I^{2}=55.1 \%\right)$. A RemL univariate meta-regression according to a country or publication year was performed, and the result indicated that only country (China/non-China) was related to the heterogeneity $(P=0.02)$, which could show $81.17 \%$ of between-study variance. In addition, we performed a sensitivity analysis, namely, the leave-one-out approach, by removing each study to compare the OR $[95 \% \mathrm{CI}]$ pooled from the remaining 12 studies with the overall OR [95\% CI] to evaluate the stability of the result. As shown in Figure 3, the result was relatively stable.

Luketich et $\mathrm{al}^{9}$ as well as Brown et $\mathrm{al}^{10}$ reported an anastomotic leak that needed surgery ( $C D$ grade $\geq 3$ ). These reports suggest that anastomotic leak requires surgical or endoscopic intervention, ${ }^{29}$ so we pooled these data and found that the incidence of severe anastomotic leak was not significantly different $(\mathrm{OR}=1.28,95 \% \mathrm{CI}=0.73-2.24, P=0.39)$, as shown in Figure 4.

\section{Other complications}

It was also revealed that MIME was associated with higher incidence of vocal cord injury/palsy $(\mathrm{OR}=5.62$,
95\% CI $=3.46-9.14, P=0.00$ ), as shown in Figure 5, as well as a higher stricture rate $(\mathrm{OR}=2.07,95 \% \mathrm{CI}=1.05-4.07$, $P=0.04)$. On the other hand, there was no evidence of significantly different incidence of Chyle leak between the 2 groups (OR $=1.42,95 \% \mathrm{CI}=0.54-3.69, P=0.48)$.

\section{Mortality}

Ten studies involving 3,034 cases addressed the 30-day/ in-hospital mortality. The mortality risk was $1.8 \%(28 / 1,537)$ in the MIME group and $1.0 \%(15 / 1,497)$ in the MILE group. As shown in Figure 6, no statistically significant difference existed between the 2 groups $(\mathrm{OR}=1.76,95 \% \mathrm{CI}=0.92-3.36$, $P=0.08)$, with statistical homogeneity $\left(I^{2}=0 \%\right)$. Additionally, 3 studies containing 499 cases reported 90-day mortality, ${ }^{21,27,28}$ and no statistically significant difference was found between the 2 groups $(\mathrm{OR}=2.22,95 \% \mathrm{CI}=0.71-6.98, P=0.17)$, as shown in Figure 7.

\section{Publication bias analysis}

Publication bias was assessed by Egger's test. Significant statistical publication bias was detected with operating time, 


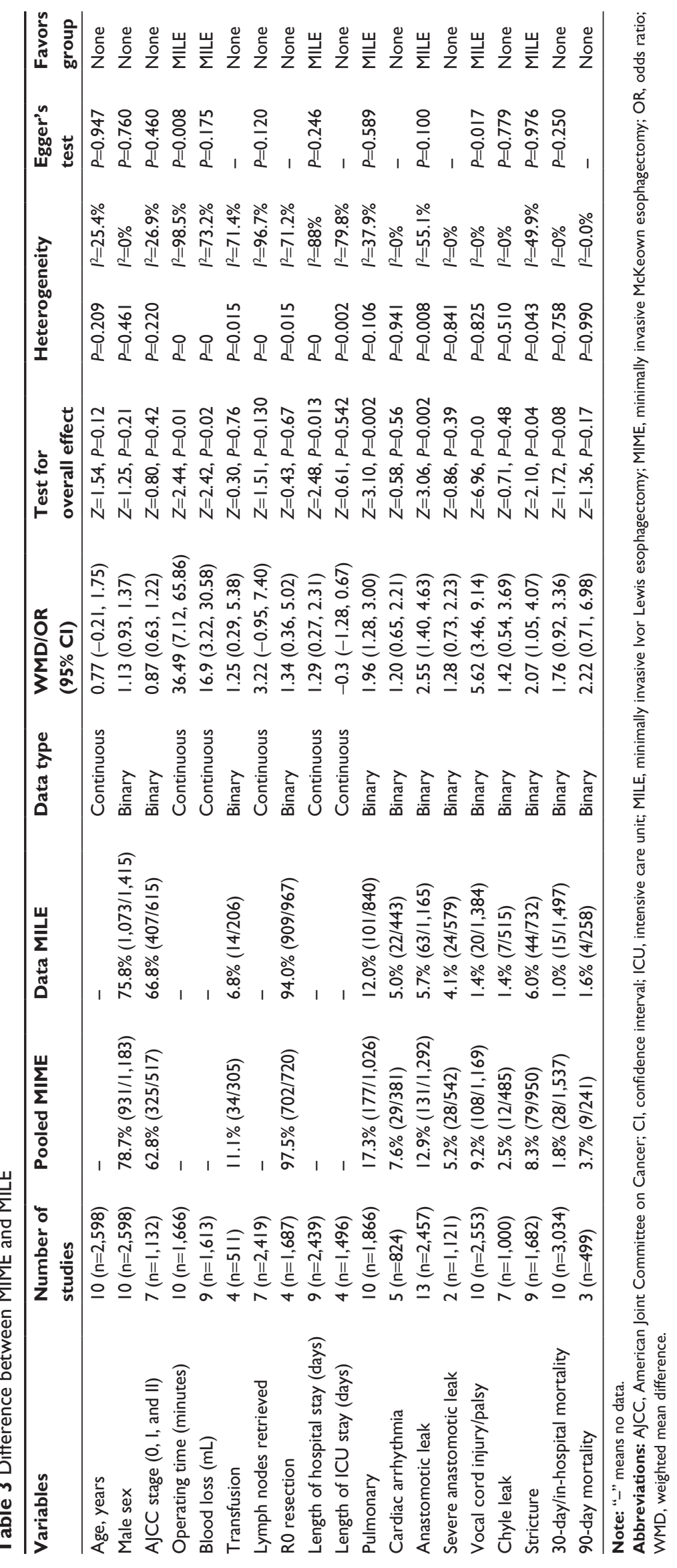




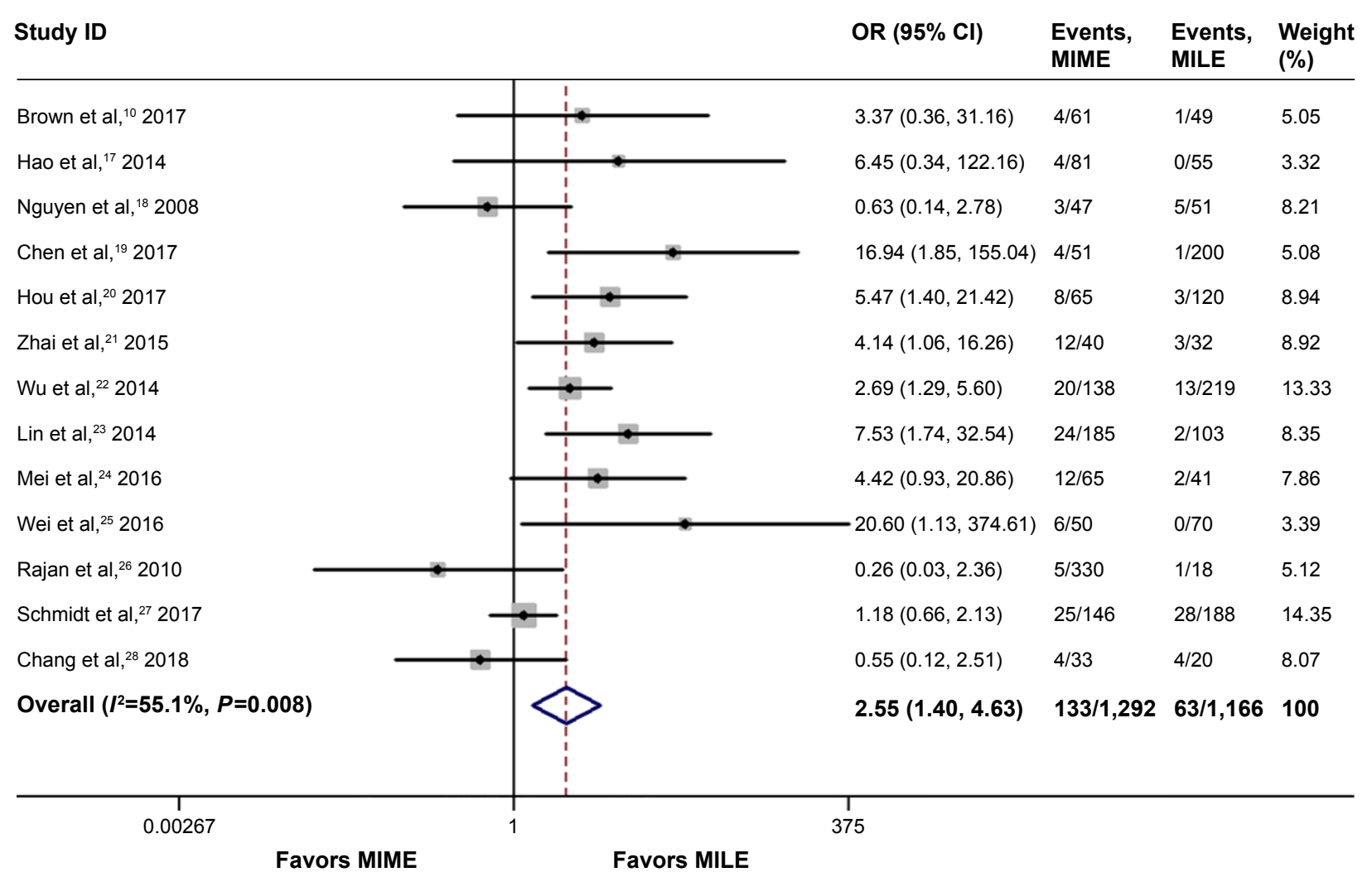

Figure 2 Meta-analysis for anastomotic leak.

Note: Weights are from random-effects analysis.

Abbreviations: $\mathrm{Cl}$, confidence interval; MILE, minimally invasive Ivor Lewis esophagectomy; MIME, minimally invasive McKeown esophagectomy; OR, odds ratio.

\section{Meta-analysis estimates, given named study is omitted}

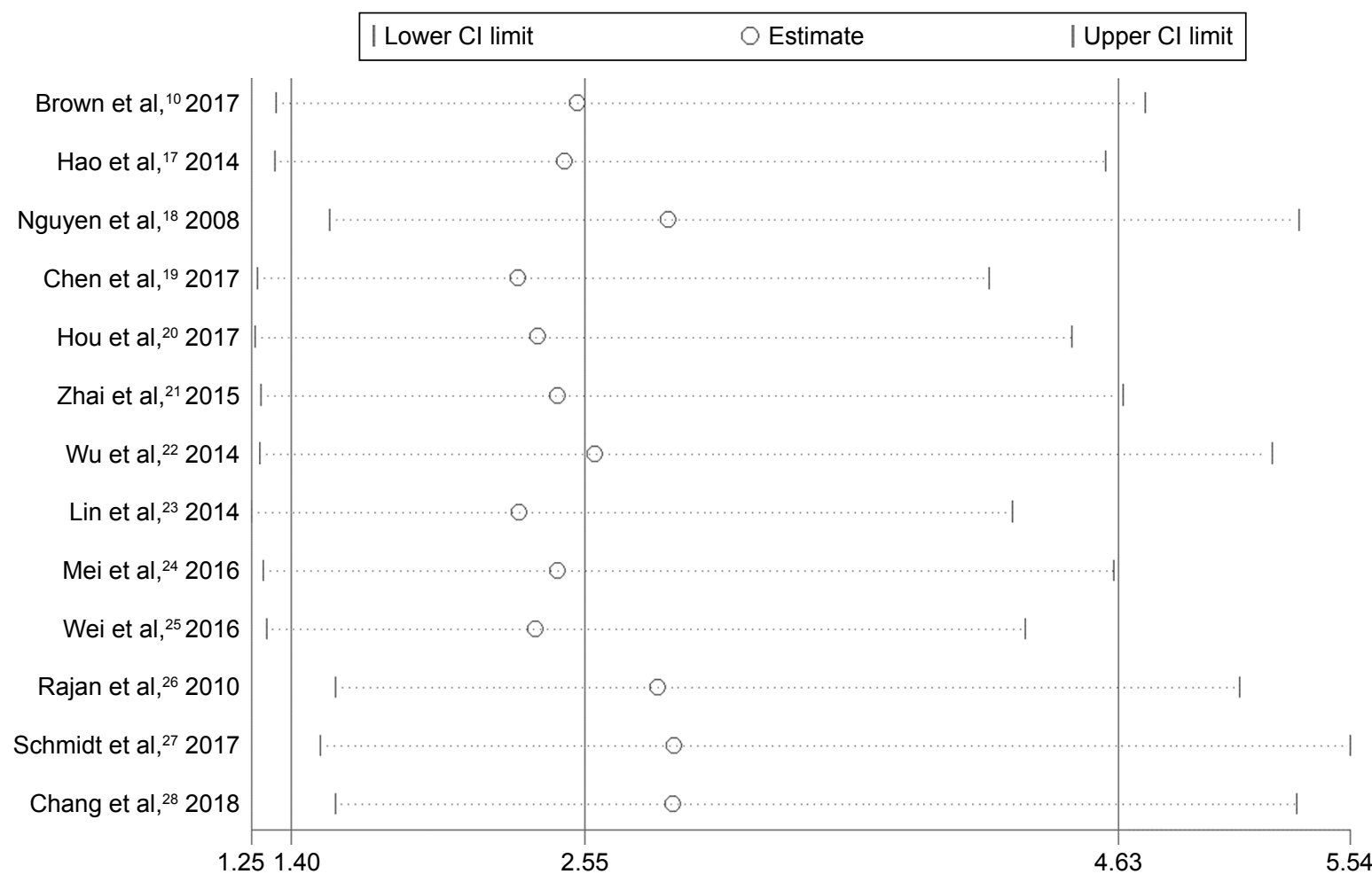

Figure 3 Sensitivity analysis for anastomotic leak.

Abbreviation: $\mathrm{Cl}$, confidence interval. 


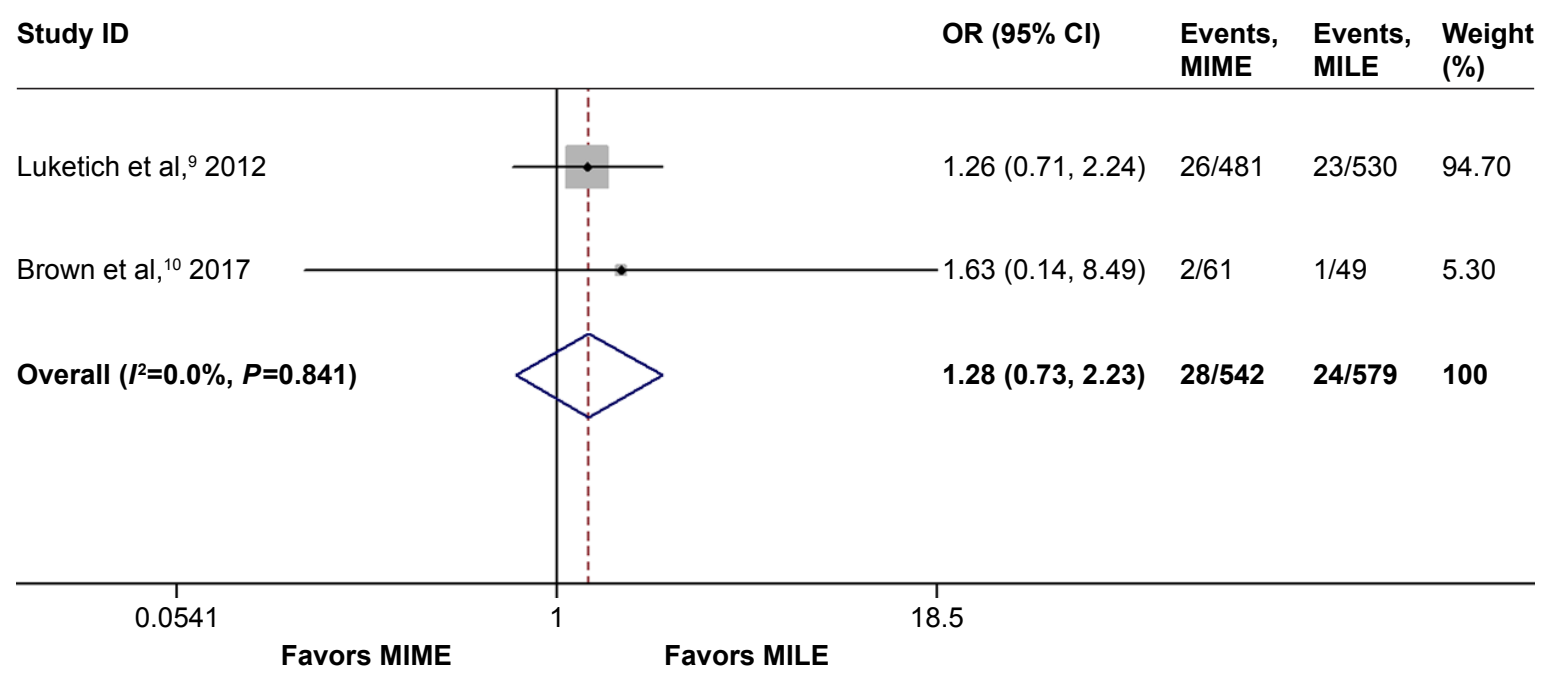

Figure 4 Meta-analysis for severe anastomotic leak.

Note: Weights are from random-effects analysis.

Abbreviations: $\mathrm{Cl}$, confidence interval; MILE, minimally invasive Ivor Lewis esophagectomy; MIME, minimally invasive McKeown esophagectomy; OR, odds ratio.

anastomotic leak, and vocal cord injury/palsy. The trimand-fill computation was carried out to estimate the effect of publication bias on the result, which indicated the result was consistent and stable.

\section{Discussion}

MILE with an intrathoracic anastomosis has become increasingly prevalent and feasible because of increasing cases of adenocarcinoma located in the distal esophagus. ${ }^{10}$ In addition, MILE was associated with lower incidence of recurrent laryngeal nerve injury and induces anastomotic leak, stricture, and swallowing dysfunction at lower rates in comparison with MIME. ${ }^{9,10}$ However, it should be noted that the results for these complications were inconsistent and MILE is not appropriate for patients with esophageal tumors located above the carina, because it might compromise

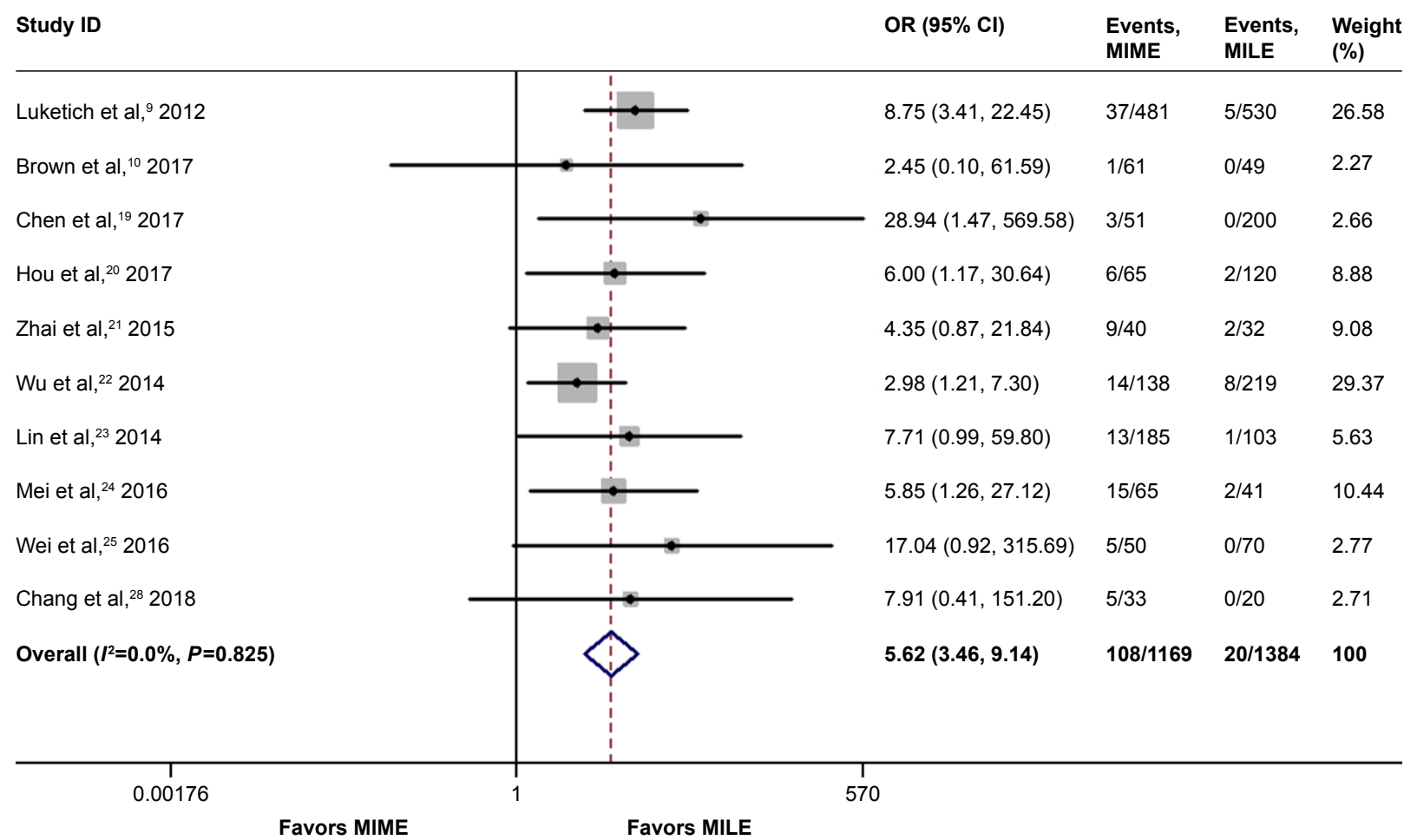

Figure 5 Meta-analysis for vocal cord injury/palsy.

Note: Weights are from random-effects analysis.

Abbreviations: $\mathrm{Cl}$, confidence interval; MILE, minimally invasive Ivor Lewis esophagectomy; MIME, minimally invasive McKeown esophagectomy; OR, odds ratio. 


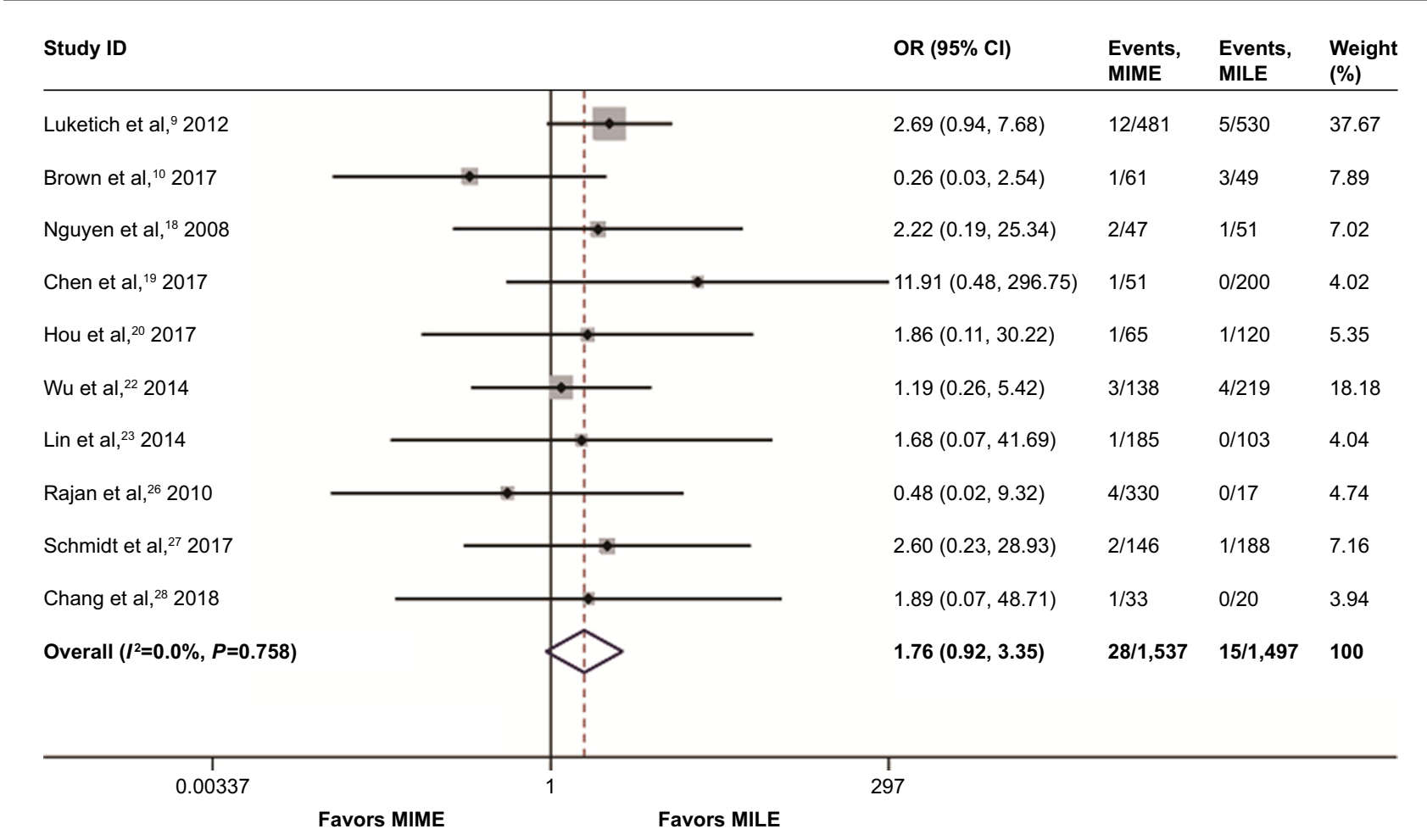

Figure 6 Meta-analysis for 30-day/in-hospital mortality.

Note: Weights are from random-effects analysis.

Abbreviations: $\mathrm{Cl}$, confidence interval; MILE, minimally invasive Ivor Lewis esophagectomy; MIME, minimally invasive McKeown esophagectomy; OR, odds ratio.

adequate resection margins. ${ }^{8}$ Potential advantages of MIME mainly include improved lymph node dissection and a more proximal resection margin. ${ }^{19}$ Additionally, MIME adopts cervical anastomosis, which is less technically challenging, and if an anastomotic leak occurs, it can be managed more easily than an intrathoracic leak. ${ }^{8}$ Therefore, it has caused a controversy about which one is preferred for patients with resectable esophageal or junctional cancer.

In the present study, we found that MIME was associated with higher rates of anastomotic leak, stricture, and pulmonary complication, and longer operating time than MILE, which were different from the findings of a previous

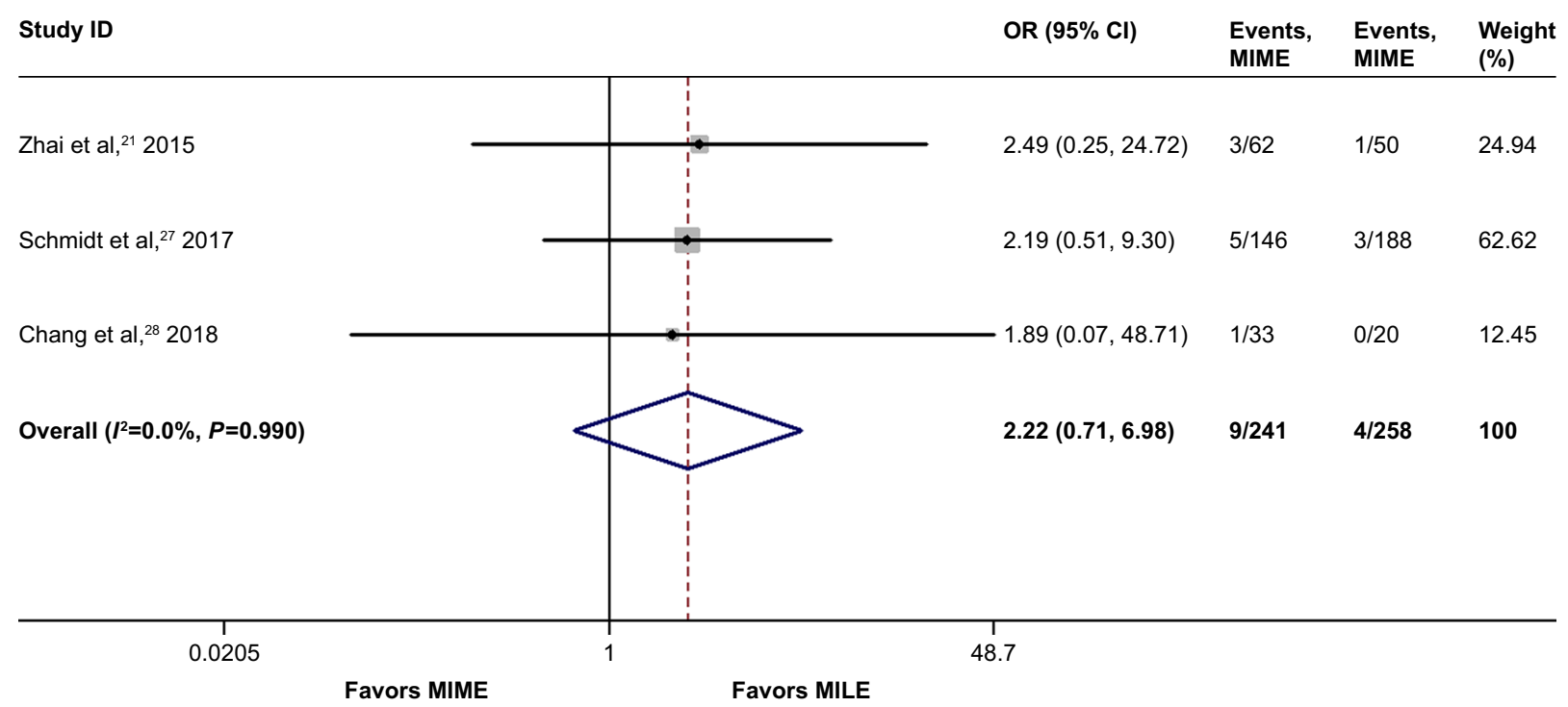

Figure 7 Meta-analysis for 90-day mortality.

Note: Weights are from random-effects analysis.

Abbreviations: $\mathrm{Cl}$, confidence interval; MILE, minimally invasive Ivor Lewis esophagectomy; MIME, minimally invasive McKeown esophagectomy; OR, odds ratio. 
meta-analysis. ${ }^{8}$ Furthermore, like the previous meta-analysis, we also found that MIME had higher incidence of vocal cord injury/palsy, more blood loss, longer length of hospital stay, equal number of lymph nodes retrieved, and similar ICU stay, similar rates of transfusion, and 30-day/in-hospital mortality compared with MILE. In addition, to our best knowledge, this is the first meta-analysis that reported similar rates of $\mathrm{R} 0$ resection, 90-day mortality, severe anastomotic leak, Chyle leak, and cardiac arrhythmia between the 2 procedures.

Because of performing an esophagogastrostomy through the third incision in the left neck, the MIME procedure will take a longer operative time and cause more blood loss. However, similar blood transfusion rates and ICU stay periods between the 2 groups was observed; transfusion of blood products in oncologic surgery is often used to determine the quality of the surgery. ${ }^{30}$ Because the number of cases included to make a pooled analysis was not large enough $(\mathrm{n}=511)$, we could not conclude whether the 2 procedures are identical in terms of surgical quality.

The oncologic efficacy was evaluated in this review. No difference in total lymph nodes retrieved during surgery and $\mathrm{R} 0$ resection rate between the 2 procedures was detected. This may be because most of the included studies adopted standard 2-field lymphadenectomy, including the left gastric and celiac nodes in patients' abdomen, as well as the periesophageal and subcarinal nodes in the chest of patients. ${ }^{10}$ In addition, MILE was performed only in the cases of esophageal cancer where clear margins can be obtained after preoperative evaluation. Combined with cautious preoperative evaluation with the clear surgical exposure provided by thoracoscopy and laparoscopy, MILE may have an equal oncologic efficacy as MIME.

After esophagectomy, anastomotic leakage is one of the most severe complications. ${ }^{31}$ Our findings showed that the MILE procedure caused less anastomotic leak than MIME, which differs from the result of the previous meta-analysis. ${ }^{8}$ This may be mainly due to the fact that MILE is able to remove the potentially ischemic gastric tip and reduce the tension at the anastomosis; thus, anastomosis can be created with a better vascularization and a lower level of the gastric tube, facilitating healing of intrathoracic anastomosis. . $^{19,32}$ In contrast, MIME requires a longer gastric tube for cervical anastomosis, which demands more vascular supply and causes higher tension and potential ischemic gastric tip existed at a higher rate at the anastomosis; also, there was oppression of thoracic outlet on the gastric tube leading to a poorly vascularized anastomosis in the neck. ${ }^{21}$ Two previous systematic reviews also reported that cervical anastomosis was associated with a higher rate of anastomotic leak. . $^{33,34}$ Significant heterogeneity was detected among studies, and meta-regression analysis found that country (China and non-China) was related to the heterogeneity. This might be because the China subgroup included 5 studies that compared MIME and MILE among esophageal cancers located in the middle and low segments. This may also be a factor that partly resulted from different anastomotic leakage between the 2 procedures. Hence, sensitivity analysis indicated that this result was stable. In addition, the 2 procedures were found to be associated with similar incidence of severe anastomotic leakage, probably because cervical leakages are easier to treat to prevent the deterioration of the leakage condition, despite higher rates in the MIME group. ${ }^{8}$ On the contrary, once the intrathoracic leakages occurs in MILE, it is much more difficult to deal with.

The incidence of anastomotic strictures was also higher in the MIME group, just as van Workum et $\mathrm{al}^{32}$ reported, it may also be caused by relatively more ischemia of the tip of the gastric tube in MIME. The 2 procedures induced chylothorax at similar rates. We found that MIME was associated with higher incidence of vocal cord injury/palsy; this may be explained by the fact that the MIME procedure necessitates a cervical dissection close to recurrent laryngeal nerve, so it is easier to cause nerve injury. ${ }^{8}$ Recurrent laryngeal nerve injury may result in aspiration and cough reflex disorders, and so MIME procedure causes more pulmonary complications. ${ }^{21}$ In addition, the 2 procedures had an equal rate of cardiac arrhythmia. Taken together, MIME was associated with higher incidence of total anastomotic leakage, stricture, vocal cord injury/palsy, and pulmonary complications, and as a result, MIME led to longer duration of hospital stay.

Despite the higher complications caused by MIME, a similar 30-day/in-hospital mortality was observed in the 2 groups, which is in concordance with the 2 latest studies. ${ }^{27,32}$ This may partly be explained by our previous result that the 2 procedures had similar incidence of causing severe anastomotic leakage. Walters et $\mathrm{a}^{35}$ suggested that 90 -day mortality may provide a better understanding on true death risk for the surgery and patient. Here, the 90-day mortality between MIME and MILE was not significantly different. Therefore, MIME and MILE are comparable with respect to clinical safety.

Compared with the previous meta-analysis in methodology, ${ }^{8}$ our study included a larger number of cases $(3,468$ vs 1,681$)$ and made a more comprehensive and rational comparison of short-term outcomes between minimally invasive McKeown and Ivor Lewis esophagectomy for 
esophageal or junctional cancer. In addition, we compared the preoperative parameters, including age, sex, and stage of the disease, and found that the 2 groups were comparable in terms of these parameters, which could increase the comparability between MIME and MILE procedures in this meta-analysis. It is worth noting that in this meta-analysis, we were not able to make a pooled analysis of other important baseline factors such as comorbidity, neoadjuvant therapy, pathology type, locations of tumors, body mass index, and smoking, all of which have been identified as risks factors for the anastomotic leak, ${ }^{36,37}$ because only few of included studies reported these parameters or because of the fact that these parameters were recorded in very different ways. This prevented us from getting a more comprehensive understanding of the comparability between the 2 groups.

There are some limitations in this meta-analysis. Some of them are as follows: 1) No randomized controlled trials were found and included in this meta-analysis; most of studies we included were retrospective cohorts, which are often subjected to considerable selection bias. 2) Definitions of outcome parameters and certain surgical details were often heterogeneous among the studies in this meta-analysis. The Esophagectomy Complications Consensus Group has proposed standardized definitions for complications after esophagectomy, which will standardize the definitions of complications in future studies. ${ }^{30}$

Overall, the present evidence indicated that MILE was associated with improved outcomes in terms of anastomotic leak, stricture, vocal cord injury/palsy, pulmonary complications, blood loss, and duration of hospital stay, but the 2 procedures had similar rates of 30-day/in-hospital and 90-day mortality, severe anastomotic leak, and oncologic efficacy. Many surgeons think that the MILE approach is only suitable for tumors located up to $5 \mathrm{~cm}$ distal of the carina, so that MILE can achieve similar oncologic efficacy as MIME does. ${ }^{38}$ Therefore, we may conclude that the 2 procedures are comparable with respect to perioperative safety. MILE may be a better option when oncologically and clinically suitable, and MIME is still a safe alternative procedure when clinically indicated.

\section{Conclusion}

In conclusion, the present meta-analysis suggests that MIME and MILE are comparable with respect to clinical safety. MILE may be a better option when oncologically and clinically suitable, and MIME is still a safe alternative procedure when clinically indicated; however, these findings are at risk for bias, and so randomized controlled trials are needed to validate or correct them.

\section{Author contribution}

Jianqing Deng, Qingqing Su, and Xiangyang Chu conceived and designed the study. Jianqing Deng, Qingqing Su, Zhipeng Ren, and Jiaxin Wen helped with the collection and assembly of data. All authors contributed toward data analysis, drafting and critically revising the paper and agree to be accountable for all aspects of the work.

\section{Disclosure}

The authors report no conflicts of interest in this work.

\section{References}

1. Parkin DM, Pisani P, Ferlay J, Statistics GC. Global cancer statistics. CA Cancer J Clin. 1999;49(1):33-64.

2. Torre LA, Bray F, Siegel RL, Ferlay J, Lortet-Tieulent J, Jemal A. Global cancer statistics, 2012. CA Cancer J Clin. 2015;65(2):87-108.

3. Gupta B, Kumar N. Worldwide incidence, mortality and time trends for cancer of the oesophagus. Eur J Cancer Prev. 2017;26(2):107-118.

4. Stahl M, Budach W, Meyer HJ, Cervantes A; ESMO Guidelines Working Group, Group EGW. Esophageal cancer: Clinical Practice Guidelines for diagnosis, treatment and follow-up. Ann Oncol. 2010;21(Suppl 5): v46-v49.

5. Biere SSAY, van Berge Henegouwen MI, Maas KW, et al. Minimally invasive versus open oesophagectomy for patients with oesophageal cancer: a multicentre, open-label, randomised controlled trial. The Lancet. 2012;379(9829):1887-1892.

6. Straatman J, van der Wielen N, Cuesta MA, et al. Minimally Invasive Versus Open Esophageal Resection: Three-year Follow-up of the Previously Reported Randomized Controlled Trial: the TIME Trial. Ann Surg. 2017;266(2):232-236.

7. Giugliano DN, Berger AC, Rosato EL, Palazzo F. Total minimally invasive esophagectomy for esophageal cancer: approaches and outcomes. Langenbecks Arch Surg. 2016;401(6):747-756.

8. van Workum F, Berkelmans GH, Klarenbeek BR, Nieuwenhuijzen GAP, Luyer MDP, Rosman C. McKeown or Ivor Lewis totally minimally invasive esophagectomy for cancer of the esophagus and gastroesophageal junction: systematic review and meta-analysis. J Thorac Dis. 2017;9(Supp1 8):S826-S833.

9. Luketich JD, Pennathur A, Awais O, et al. Outcomes after minimally invasive esophagectomy: review of over 1,000 patients. Ann Surg. 2012; 256(1):95-103.

10. Brown AM, Pucci MJ, Berger AC. A standardized comparison of peri-operative complications after minimally invasive esophagectomy: Ivor Lewis versus McKeown. Surg Endosc. 2017;32(1):204-211.

11. He J, Shao K. The epidemiology, current status of management, challenge and future strategy for esophageal cancer in China. China Oncology. 2011;21(7):501-504.

12. Higgins J, Green S. Cochrane Handbook for Systematic Reviews of Interventions Version 5.1.0 [updated March 2011]. London: The Cochrane Collaboration; 2011. Available from: http://handbook-5-1. cochrane.org/. Accessed September 10, 2018.

13. Liberati A, Altman DG, Tetzlaff J, et al. The PRISMA statement for reporting systematic reviews and meta-analyses of studies that evaluate healthcare interventions: explanation and elaboration. $B M J$. 2009;339:b2700.

14. Wells GA, Shea B, O'Connell D, Peterson J, Welch V, Losos M, et al. The Newcastle-Ottawa scale (NOS) for assessing the quality of nonrandomised studies in meta-analyses. Ottawa Hospital Research Institute; 2014. Available from: http://www.ohri.ca/programs/clinical_epidemiology/ oxford.asp. Accessed August 15, 2017.

15. Zeng X, Ren X. To Perform Meta-Analysis with Stata Software. Vol 2. Beijing: Peking Union Medical College Press; 2017:4. 
16. Zuo J WQ, Zhou A, Zhou J, Song Y, Xu G. The short-term outcomes of Ivor Lewis esophagectomy versus McKeown esophagectomy for thoracic middle-lower esophageal carcinoma: a systematic review and meta-analysis. Chinese Journal of Clinical Thoracic and Cardiovascular Surgery. 2017;2(6):456-462.

17. Hao Z, Zhenya S, Lei W, Zhang H, Shen Z, Wang L. Esophagealgastric anastomosis in radical resection of esophageal cancer under thoracoscopy combined with laparoscopy. J Coll Physicians Surg Pak. 2014;24(10):754-756.

18. Nguyen NT, Hinojosa MW, Smith BR, Chang KJ, Gray J, Hoyt D. Minimally invasive esophagectomy: lessons learned from 104 operations. Ann Surg. 2008;248(6):1081-1091.

19. Chen L, Liu X, Wang R, et al. Minimally invasive esophagectomy for esophageal cancer according to the location of the tumor: Experience of 251 patients. Ann Med Surg. 2017;17:54-60.

20. Hou X, Ren Z, Yang B, Liu Y. Comparison of short-term therapeutic efficacy between minimally invasive Ivor-Lewis esophagectomy and Mckeown esophagectomy for esophageal cancer. Biomedical Research. 2017;28(12):5321-5326.

21. Zhai C, Liu Y, Li W, et al. A comparison of short-term outcomes between Ivor-Lewis and McKeown minimally invasive esophagectomy. J Thorac Dis. 2015;7(12):2352-2358.

22. Wu H, Xie M, Liu C, Xu M, Guo M. A retrospedive study of shortterm outcomes between minimally invasive McKeown esophagectomy and Lvor-Lewis esophagectomy for throcic middle-lower esphageal carcinoma. Chin J Thorac Cardiovasc Surg. 2014;30(11):649-652.

23. Lin J, Kang M, Lin J, et al. The short-term efficacy comparison between Ivor-Lewis esophagectomy approach and McKeown approach in minimally invasive esophagectomy. Chin J Gastrointest Surg. 2014; 17(9):888-891.

24. Mei S, Liu J, Wu H, Wu D, Xie Y, Mou Z. Short-term efficacy of purse sting forceps Ivor-Lewis approach and McKeown approach in minimally invasive esophagectomy for middle-lower section esophageal cancer. Jounral of Chongqing Medical University. 2016;1:89-93.

25. Wei H, Wang Z, et al. The comparision of clinical curative effect of IvorLewis and McKeown operation in totally endoscopic esophagectomy. Chinese Journal of Clinical Thoracic and Cardiovascular Surgery. 2016;32(8):495-498.

26. Rajan PS, Vaithiswaran V, Rajapandian S, Senthilnathan P, Praveenraj P, Palanivelu C. Minimally invasive oesophagectomy for carcinoma oesophagus - approaches and options in a high volume tertiary centre. J Indian Med Assoc. 2010;108(10):642-644.
27. Schmidt HM, Gisbertz SS, Moons J, et al. Defining Benchmarks for Transthoracic Esophagectomy: A Multicenter Analysis of Total Minimally Invasive Esophagectomy in Low Risk Patients. Ann Surg. 2017;266(5):814-821.

28. Chang T, Hsiao PN, Tsai MY, Huang PM, Cheng YJ. Perioperative management and outcomes of minimally invasive esophagectomy: case study of a high-volume tertiary center in Taiwan. J Thorac Dis. 2018; 10(3):1670-1676.

29. Dindo D, Demartines N, Clavien P-A. Classification of Surgical Complications. Ann Surg. 2004;240(2):205-213.

30. Low DE, Alderson D, Cecconello I, et al. International Consensus on Standardization of Data Collection for Complications Associated With Esophagectomy: Esophagectomy Complications Consensus Group (ECCG). Ann Surg. 2015;262(2):286-294.

31. Morita M, Nakanoko T, Fujinaka Y, et al. In-hospital mortality after a surgical resection for esophageal cancer: analyses of the associated factors and historical changes. Ann Surg Oncol. 2011;18(6):1757-1765.

32. van Workum F, van der Maas J, van den Wildenberg FJ, et al. Improved Functional Results After Minimally Invasive Esophagectomy: Intrathoracic Versus Cervical Anastomosis. Ann Thorac Surg. 2017; 103(1):267-273.

33. Biere SS, Maas KW, Cuesta MA, van der Peet DL. Cervical or thoracic anastomosis after esophagectomy for cancer: a systematic review and meta-analysis. Dig Surg. 2011;28(1):29-35.

34. Rindani R, Martin CJ, Cox MR, Walther B, Johansson J, Johnsson F. Transhiatal versus Ivor-Lewis oesophagectomy: is there a difference? Aust N Z J Surg. 1999;69(3):187-194.

35. Walters DM, Mcmurry TL, Isbell JM, Stukenborg GJ, Kozower BD. Understanding mortality as a quality indicator after esophagectomy. Ann Thorac Surg. 2014;98(2):506-512.

36. Chen C, Yu Z, Jin Q, Zhang X. Clinical features and risk factors of anastomotic leakage after radical esophagectomy. Zhonghua Wai Ke Za Zhi. 2015;53(7):518-521.

37. van Daele E, van de Putte D, Ceelen W, van Nieuwenhove Y, Pattyn P. Risk factors and consequences of anastomotic leakage after Ivor Lewis oesophagectomy. Interact Cardiovasc Thorac Surg. 2016;22(1):32-37.

38. Straatman J, van der Wielen N, Nieuwenhuijzen GAP, et al. Techniques and short-term outcomes for total minimally invasive Ivor Lewis esophageal resection in distal esophageal and gastroesophageal junction cancers: pooled data from six European centers. Surg Endosc. 2017;31(1):119-126.
OncoTargets and Therapy

\section{Publish your work in this journal}

OncoTargets and Therapy is an international, peer-reviewed, open access journal focusing on the pathological basis of all cancers, potential targets for therapy and treatment protocols employed to improve the management of cancer patients. The journal also focuses on the impact of management programs and new therapeutic agents and protocols on

\section{Dovepress}

patient perspectives such as quality of life, adherence and satisfaction. The manuscript management system is completely online and includes a very quick and fair peer-review system, which is all easy to use. Visit http://www.dovepress.com/testimonials.php to read real quotes from published authors. 